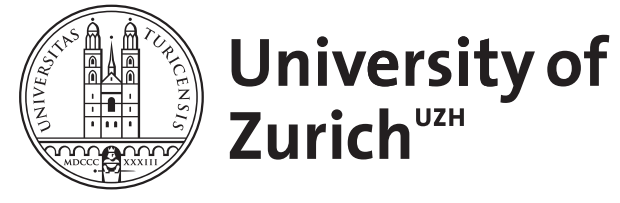

Note: Deep UV-pump THz-probe spectroscopy of the excess electron in water

\author{
Berger, Arian ; Savolainen, Janne ; Shalit, Andrey ; Hamm, Peter
}

DOI: https://doi.org/10.1063/1.4991462

Posted at the Zurich Open Repository and Archive, University of Zurich ZORA URL: https://doi.org/10.5167/uzh-150198

Journal Article

Published Version

Originally published at:

Berger, Arian; Savolainen, Janne; Shalit, Andrey; Hamm, Peter (2017). Note: Deep UV-pump THz-probe spectroscopy of the excess electron in water. Journal of Chemical Physics, 146(24):246101.

DOI: https://doi.org/10.1063/1.4991462 
Note: Deep UV-pump THz-probe spectroscopy of the excess electron in water

Arian Berger, Janne Savolainen, Andrey Shalit, and Peter Hamm

Citation: The Journal of Chemical Physics 146, 246101 (2017); doi: 10.1063/1.4991462

View online: https://doi.org/10.1063/1.4991462

View Table of Contents: http://aip.scitation.org/toc/jcp/146/24

Published by the American Institute of Physics

\section{Articles you may be interested in}

Perspective: Echoes in 2D-Raman-THz spectroscopy

The Journal of Chemical Physics 146, 130901 (2017); 10.1063/1.4979288

Broadband terahertz pulse generation by optical rectification in GaP crystals

Applied Physics Letters 110, 201103 (2017); 10.1063/1.4983371

Mass density fluctuations in quantum and classical descriptions of liquid water

The Journal of Chemical Physics 146, 244501 (2017); 10.1063/1.4986284

IR spectral assignments for the hydrated excess proton in liquid water

The Journal of Chemical Physics 146, 154507 (2017); 10.1063/1.4980121

Hydrogen bonding characterization in water and small molecules

The Journal of Chemical Physics 146, 244315 (2017); 10.1063/1.4990504

Molecule-specific interactions of diatomic adsorbates at metal-liquid interfaces

Structural Dynamics 4, 044009 (2017); 10.1063/1.4978894

\section{AIP | The Journal of Chemical Physics}




\title{
Note: Deep UV-pump THz-probe spectroscopy of the excess electron in water
}

\author{
Arian Berger, Janne Savolainen, Andrey Shalit, and Peter Hamm \\ Department of Chemistry, University of Zurich, Zurich, Switzerland
}

(Received 5 May 2017; accepted 20 June 2017; published online 30 June 2017)

[http://dx.doi.org/10.1063/1.4991462]

In a previous paper, ${ }^{1}$ we have shown that the excess (hydrated) electron in water, at the initial moment of its generation by photo-detachment, occupies a delocalized state with a size of at least $40 \AA$ upon photoionization at $800 \mathrm{~nm}$. According to a simple particle-in-a-box picture, the s-p transition of such a large electron wavefunction occurs at around $1.5 \mathrm{THz}$, which is what has been observed in Ref. 1. On a time scale of $\sim 200 \mathrm{fs}$, the absorption leaves the $\mathrm{THz}$ observation window as the electron wavefunction gets solvated, collapses, and eventually shifts into the near-infrared (NIR) range, where the electron is stable on a nano- to micro-second time scale in neat water. It has also been suggested in Ref. 1 that the initial delocalization length of the excess electron correlates well with the ejection distance of the collapsed wavefunction after solvation. The latter can be determined via the rate of the diffusive kinetics of geminate recombination. ${ }^{2-4}$

In this note, we extended the series of the excitation wavelengths from Ref. 1, i.e., $800 \mathrm{~nm}, 400 \mathrm{~nm}$, and $266 \mathrm{~nm}$, down to the deep UV at $200 \mathrm{~nm}$. It is generally accepted that there are at least two photoionization pathways for the excess electron. $2,3,5,6$ That is, if the excitation energy is large enough $(>9.8-9.9 \mathrm{eV}),{ }^{3}$ the electron can reach the conduction band of water directly and vertically. At energies below, a concerted nuclear rearrangement or a coincidentally pre-arranged minimum on the potential surface are required for the detachment process to occur. In the cases of $400 \mathrm{~nm}$ and $266 \mathrm{~nm}$ excitation wavelengths, at least 3-4 photons are needed to reach the direct ionization threshold; hence, the detachment process probably is a mixture of both mechanisms and the interpretation becomes more complicated. On the other hand, two-photon absorption with $200 \mathrm{~nm}$ pulses (see Fig. 1, inset) amounts to $12.4 \mathrm{eV}$, clearly exceeding that threshold, while the single-photon absorption cross section is negligible.

For the most part, we discussed $800 \mathrm{~nm}$ excitation in Ref. 1, which so far has hardly been considered in the literature as a way to produce excess electrons in water. ${ }^{7,8}$ In that case, an irradiance threshold exists with excess electrons being formed only above $\sim 1 \mathrm{TW} / \mathrm{cm}^{2}$, indicating a field ionization process for the detachment rather than multi-photon absorption. ${ }^{1,8}$ It is thus difficult to assign an excitation energy to the process, since one can no longer count the number of absorbed photons. Nevertheless, the recombination kinetics gave an indication for the effective excitation energy. That is, the geminate recombination kinetics reflects the diffusive process of the hydrated electron, which takes longer if the electron is further away from its hole. ${ }^{2,3}$ Consequently, the time scale of the recombination kinetics is a measure of the initial ejection distance of the electron. We found in Ref. 1 that the recombination kinetics after $800 \mathrm{~nm}$ pumping is essentially the same as that after $200 \mathrm{~nm}$ pumping; ${ }^{3,4}$ Fig. 1 makes that comparison explicit. This indicates that the effective excitation energy and thus the excitation pathway are the same in both cases. On the other hand, both are distinctively different from $400 \mathrm{~nm}$ to $266 \mathrm{~nm}$ pumping, where the recombination rate is faster ${ }^{1,3}$ and thus the ejection distance is smaller.

However, the recombination kinetics is only a very indirect manifestation of the detachment mechanism, which is why we set out here to measure the $\mathrm{THz}$ response following $200 \mathrm{~nm}$ pumping. The $\mathrm{THz}$ absorption spectrum of the excess electron directly reflects its initial delocalization length. ${ }^{1}$ To that end, $200 \mathrm{~nm}$ pulses have been generated in a similar fashion as in Ref. 10 but with type I phase matching in the THG step $(\theta=44.3,0.3 \mathrm{~mm})$. The pulse energy at $200 \mathrm{~nm}$ was $2.8 \mu \mathrm{J}$ and the pulse duration was $150 \mathrm{fs}$ (see Fig. 2, inset). The THzpart of the experimental setup was the same as in Ref. 1 with single cycle THz-probe pulses centered at $\sim 1.5 \mathrm{THz}$.

Figure 2 compares the experimental results for $200 \mathrm{~nm}$ and $800 \mathrm{~nm}$ pumping. The two data sets were scaled by normalization of the residual signal at 2 ps (see blow-up in Fig. 2). In the case of $800 \mathrm{~nm}$ pumping, this long term signal was shown to stay constant up to $300 \mathrm{ps}^{1}$ and thus was assigned to a rise in temperature in the sample. ${ }^{11}$ Consequently, the residual signal is proportional to the energy deposited into the sample and thus should also be proportional to the amount of detached electrons, at least when assuming that the detachment pathways are the same. In both cases, a spike around a pump-probe delay of $\tau=0$ is observed (somewhat larger for $200 \mathrm{~nm}$ pumping), whose width reflects the cross-correlation of pump and probe pulses (to that end, compare to the pump-pulse autocorrelation functions shown in the inset of Fig. 2). This feature is probably caused by an induced multi-photon absorption process of the $\mathrm{THz}$ probe pulse.

The signal after the pump-probe overlap starting at $\sim 200$ fs contains the information on the delocalized electron. As can be seen, the $\mathrm{THz}$ signal decays basically identically in both cases, with an exponential fit revealing a decay time of $\approx 170 \mathrm{fs}$ in either case. According to the clean two-photon process (Fig. 1, inset), the $200 \mathrm{~nm}$ photoionization process is known with certainty to produce excess electrons via vertical transition into the conduction band. The perfect agreement of the $\mathrm{THz}$ responses following $800 \mathrm{~nm}$ pumping suggests that 


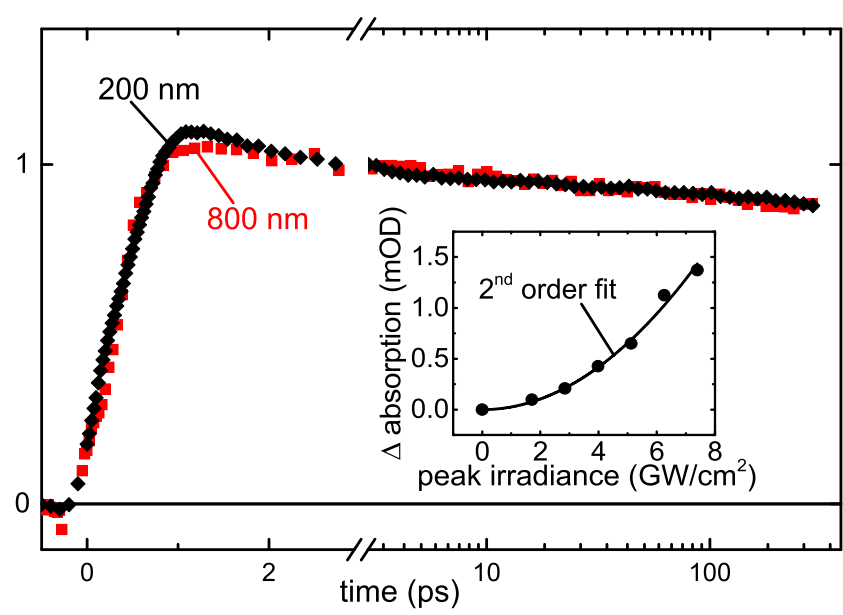

FIG. 1. Recombination kinetics after pumping at $200 \mathrm{~nm}$ (black) or $800 \mathrm{~nm}$ (red, the latter taken from the supplementary material of Ref. 1). In both cases, the solvated electron is probed at $800 \mathrm{~nm}$. The data are normalized at 3 ps after excitation, when the excess electron is fully solvated and equilibrated. The inset shows the power dependence after $200 \mathrm{~nm}$ pumping together with a quadratic fit, indicating a two-photon absorption process.

the initial size of the delocalized electron is the same. This correlation furthermore indicates that the ejection mechanism as well as the effective excitation energy of $12.4 \mathrm{eV}$ upon 800 $\mathrm{nm}$ and $200 \mathrm{~nm}$ pumping is also the same, at least for the experimental condition with which we performed our experiments (one might assume that the effective excitation energy in a field-ionization process depends on the pump intensity; in both Figs. 1 and 2, the peak intensity of $800 \mathrm{~nm}$ pumping was $\sim 5 \mathrm{TW} / \mathrm{cm}^{2}$ ).

Pumping with $200 \mathrm{~nm}$ provides a much cleaner way to generate the excess electron directly in the conduction band. The $a b$ initio MD simulation of Ref. 1 implicitly assumed that detachment pathway by artificially adding an excess electron without any nuclear rearrangement at time 0 . Consequently, the conclusions of Ref. 1 also apply to the dynamics of the excess electron following pumping at $200 \mathrm{~nm}$.

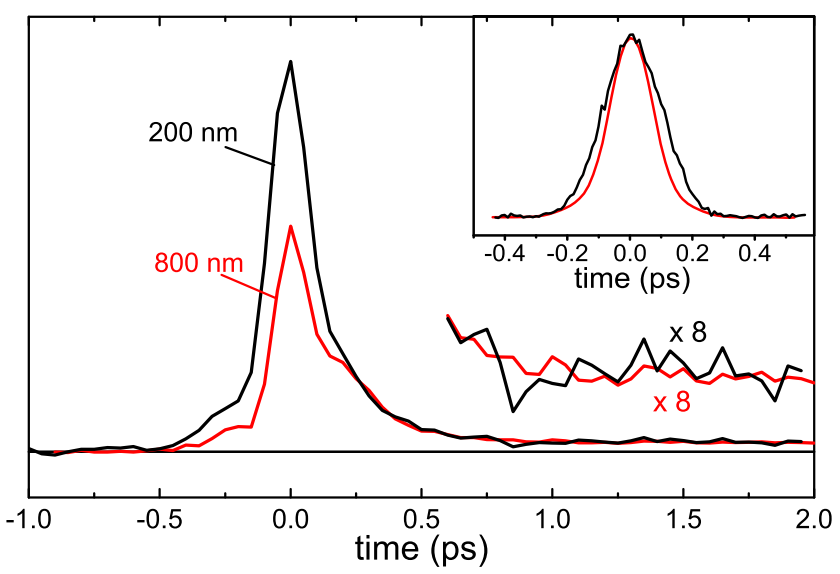

FIG. 2. THz response of the excess electron after $200 \mathrm{~nm}$ pumping (black) and $800 \mathrm{~nm}$ pumping (red). The $800 \mathrm{~nm}$ data are, in principle, the same as in Ref. 1 but have been remeasured for reasons of consistency. Both signals are normalized to the residual heat signal at 2 ps. The inset shows the autocorrelation of the pump pulses, which in the case of $200 \mathrm{~nm}$ was measured as described in Ref. 9.

We thank Pavel Jungwirth for many insightful discussions on the topic. The work has been supported by the Swiss National Science Foundation (SNF) through the NCCR MUST.

${ }^{1}$ J. Savolainen, F. Uhlig, S. Ahmed, P. Hamm, and P. Jungwirth, Nat. Chem. 6, 697 (2014).

${ }^{2}$ R. Laenen and T. Roth, J. Mol. Struct. 598, 37 (2001).

${ }^{3}$ C. G. Elles, A. E. Jailaubekov, R. A. Crowell, and S. E. Bradforth, J. Chem. Phys. 125, 044515 (2006).

${ }^{4}$ R. Lian, D. A. Oulianov, I. A. Shkrob, and R. A. Crowell, Chem. Phys. Lett. 398, 102 (2004).

${ }^{5}$ M. U. Sander, K. Luther, and J. Troe, Ber. Bunsengesellschaft Phys. Chem. 97, 953 (1993).

${ }^{6}$ R. A. Crowell and D. M. Bartels, J. Phys. Chem. 100, 17940 (1996).

${ }^{7}$ M. S. Brown, T. Erickson, K. Frische, and W. M. Roquemore, Opt. Express 19, 12241 (2011).

${ }^{8}$ J. Li, Z. Nie, Y. Y. Zheng, S. Dong, and Z.-H. Loh, J. Phys. Chem. Lett. 4, 3698 (2013).

${ }^{9}$ C. Homann, N. Krebs, and E. Riedle, Appl. Phys. B 104, 783 (2011).

${ }^{10}$ J. Ringling, F. Noack, G. Korn, and J. Squier, Opt. Lett. 18, 2035 (1993).

${ }^{11}$ H. R. Zelsmann, J. Mol. Struct. 350, 95 (1995). 